\title{
Le Programme WATSAVE de la CIID
}

\author{
par Alain Vidal \\ Président du Comité technique de l'AFEID \\ Cemagref, Division Irrigation
}

\section{CONTEXTE ET OBJECTIFS}

D'ici la fin du siècle, la population mondiale dépassera les 6 milliards dont $90 \%$ se trouveront dans les pays en développement, et on prévoit que la poussée démographique pourrait devancer l'accroissement de la production vivrière. Au cours des quatre dernières décennies, l'irrigation et le drainage ont apporté une contribution importante à l'accroissement de la production alimentaire : actuellement, $1 / 6$ seulement des terres agricoles irriguées produit $1 / 3$ des cultures vivrières, et le drainage a augmenté la productivité d'encore un dixième de la superficie cultivée.

Cependant, l'agriculture irriguée consomme de 70 à $80 \%$ de l'eau douce utilisée dans les pays en développement. La performance de nombreux projets d'irrigation est au-dessous de leur niveau potentiel: sur le plan mondial, près des deux cinquièmes ( 100 millions d'hectares) des terres irriguées sont déjà incultivables, ou risquent de le devenir, en raison de phénomènes de remontées de nappe ou de salinité. Toute croissance future de l'agriculture irriguée pourrait être limitée par la pénurie d'eau et de terres, par une concurrence accrue entre les différents usages de l'eau, par une mauvaise gestion dont le corollaire est la détérioration des systèmes existants.

Le Sommet de la Terre (Rio de Janeiro, juin 1992) a lancé des défis redoutables au développement des ressources en eau et à la production agricole. Le Plan d'Action $21 \mathrm{du}$ Sommet de la Terre prévoit une cible de 3 à $4 \%$ par an pour un accroissement durable de la production vivrière, y compris la croissance de la productivité découlant des ouvrages d'irrigation existants. Cette cible empêchera ou même renversera la dégradation future des ressources en eau et en terre.

La Commission Internationale des Irrigations et du Drainage (CID) a acquis, au long d'un demi-siècle, une expérience significative en matière de recherche, de développement et de transfert des technologies de gestion de l'eau. Lors de son $15^{\text {e }}$ Congrès à La Haye, en 1992, et dans la suite du Sommet de la Terre, la CIID a mis en place les actions suivantes:

1. Favoriser de nouveaux programmes visant à des économies d'eau dans l'agriculture afin que l'eau soit disponible pour d'autres usages prioritaires émergents ;

2. Encourager les agences d'irrigation et de drainage à optimiser l'utilisation des ressources et à adopter des approches holistiques et multidisciplinaires de développement des systèmes d'irrigation et de drainage, lesquels constituent, dans une large mesure, la clé de réalisation de résultats durables ;

3. Favoriser les programmes destinés à accroître la productivité de l'eau, tant au niveau de l'exploitation qu'à celui du périmètre, et à assurer la répartition équitable de l'eau, le développement durable et la protection de l'environnement ; 4. Lancer, en association avec d'autres agences, des campagnes pour sensibiliser le public et encourager sa participation à l'occasion de la Journée Mondiale de l'Eau, fixée chaque année au 22 mars, conformément à la décision des Nations Unies ;

\section{The CIID WATSAVE program}

In order to face the challenges given to irrigated agriculture by the Earth Summit of Rio in 1992, the International Commission on Irrigation and Drainage (ICID) has defined major priority actions, whose first is the Watsave plan for water conservation. This programme, based on an experts team representing main irrigating countries of the world, aims at providing recommendations for water conservation from experience acquired in these countries. A first questionnaire has already permitted to set priorities to be pronioted: public sensitisation, development of adapted water tariffs, integrated management of water resources, improvement of irrigation techniques, monitoring of environmental impacts, wastewater reuse. 
5. Inciter par le biais des agences d'irrigation et de drainage les organisations d'agriculteurs à participer plus activement à l'exploitation, à l'entretien et à la gestion des systèmes d'irrigation et de drainage ;

6. Promouvoir la coopération internationale dans l'aménagement des bassins fluviaux internationaux;

7. Favoriser des programmes spécifiques dans les domaines de l'irrigation, du drainage et de la gestion d'eau en Afrique et dans d'autres régions touchées par des pénuries d'eau;

8. Elaborer des projets axés sur des zones spécifiques pour faire face aux sécheresses et aux inondations;

9. Renforcer les adhésions à la CIID et à ses comités nationaux et former des jeunes professionnels ;

10. Entreprendre des programmes d'échanges technolo giques entre Comités nationaux, planificateurs et gestionnaires des systèmes d'irrigation.

A la suite de ces décisions, la CID a mis en place le programme WATSAVE ou Plan d'Action Global pour la Conservation de l'Eau. Les travaux ont été confiés à une équipe de travail présidée par le Président sortant John Hennessy, comportant des représentants de la Chine, de l'Inde, du Pakistan, de l'Egypte, du Maroc et du Mexique. Se sont ensuite joints à cette équipe l'Afrique du Sud, l'Australie, la France, Israël et les Etats-Unis. Les pays représentés possèdent ainsi $50 \%$ des surfaces irriguées dans le monde.

Les objectifs de WATSAVE sont les suivants :

- promouvoir la conservation de l'eau dans les pays membres de la CIID comme une composante de la gestion de l'eau:

- comprendre et comparer les programmes de conservation de l'eau des pays membres pour en tirer des enseignements - fournir des recommandations aux pays membres sur des programmes nationaux et régionaux de conservation de l'eau ; - promouvoir le partenariat entre pays membres ;

- identifier et faire connaître les expériences réussies de conservation de l'eau.

WATSAVE s'appuie sur trois composantes

- l'équipe WATSAVE, qui fera connaître les activités des pays représentés en matière de conservation de l'eau, de façon à permettre aux autres pays membres de la CIID de mettre en place leur propre plan de conservation de l'eau ;

- la diffusion auprès des pays membres d'un questionnaire détaillé sur les mesures de conservation de l'eau:

- la préparation d'un rapport WATSAVE sur la conservation de l'eau résultant de l'analyse des réponses au questionnaire détaillé, et qui sera présenté au $16^{\mathrm{C}}$ Congrès CIID du Caire en 1996 afin de formuler un futur plan d'action CID.

\section{PREMIER QUESTIONNAIRE WATSAVE}

Un premier questionnaire simplifié diffusé en 1994 aux pays représentés dans WATSAVE a permis de dresser un premier tableau des mesures et des politiques de conservation de l'eau. Ce questionnaire était le suivant

1. Existe-t-il des directives nationales concernant la conservation de l'eau? Spécifier.

2. Situation actuelle des ressources.

3. Mesures de conservation de l'eau (aux échelles de

l'exploitation, régionale et nationale).

4. Quantité et qualité de l'eau disponible.

5. Besoins en eau et mesures de conservation de l'eal des autres usagers de l'eau (domestiques, municipaux, industriels et de loisir).

6. Existe-t-il des programmes nationaux? Contenu et objectifs?

7. Enseignements tirés des mesures de conservation de l'eau.

8. Futurs programmes nationaux et régionaux, en particulier quels besoins en matière d'aide à la décision?

9. Conclusions et faits marquants.
L'Australie, la Chine, la France, l'Inde, Isräl, le Pakistan, l'Afrique du Sud et les Etats-Unis ont répondu à ce questionnaire. L'analyse et la synthèse des réponses font l'objet d'un livret en anglais édité par la CID : "WATSAVE Activities, some lessons and experiences ", dont nous reprendrons ici les principales conclusions.

\section{CONCLUSIONS DU PREMIER QUES- TIONNAIRE WATSAVE}

On a démontré que les campagnes de publicité sont nécessaires et efficaces. Le public répond favorablement aux conseils d'économie d'eau, en réduisant sa consommation, en particulier lorsque la tension apparaît clairement, comme en Israël. Le public coopère et s'équipe en conséquence.

Une augmentation du prix de l'eau et un soutien financier facilitent les actions de réhabilitation et d'amélioration des réseaux, réduisant ainsi les pertes. La tarification joue un rôle important pour rendre la conservation de l'eau efficace. La mise en place de lois sur l'eau, comme en France et en Australie, sont nécessaires pour une gestion intégrée de l'eau, et une politique centrale de promotion d'une utilisation rationnelle de l'eau est nécessaire et utile, comme cela a été démontré en Israël. Le contrôle des prélèvements apparaît comme essentiel.

Le concept de «propriété communautaire (community ownership) de l'eau, par exemple à l'échelle d'un bassin versant, permet d'assurer une utilisation durable de l'eau et des ressources naturelles par tous les usagers d'une communauté, à l'image des ICM (Integrated Catchment Management) australiens, ou systèmes de gestion intégrée de bassins versants. La tendance générale de transfert de la gestion de l'eau aux usagers doit les impliquer beaucoup plus dans les mesures de conservation de l'eau.

Les améliorations des techniques d'irrigation permettant de réduire les consommations en eau ne doivent pas être négligées sur la simple base de leur surcoût: les centres de recherche et de formation doivent permettre de diffuser plus largement ces améliorations. La mise en place de cellules de pilotage de l'irrigation au sein des organismes chargés de gérer l'irrigation permet une conservation de l'eau efficace, comme l'a démontré le Pakistan. La mise en place de réseaux d'établissements de recherche en irrigation permet de sensibiliser et de former les professionnels aux techniques de conservation de l'eau.

Les besoins en eau pour l'environnement (débits réservés, protection des zones humides, biodiversité) pèsent sur la disponibilité en eau. Par ailleurs, la détérioration de la qualité de l'eau (salinisation, pollutions diffuses) nécessite un suivi renforcé. Enfin, le traitement et la réutilisation des eaux usées constituent une composante importante de la conservation de l'eau, et s'imposent parfois, comme en Israël, comme une nécessité.

\section{BIBLIOGRAPHIE}

\section{[1] Bulletin CGGREF.}

[2] ICID-CID, 1995. - Watsave activities: some lessons and experiences. CID. New Delhi, 1995, 32 p. 